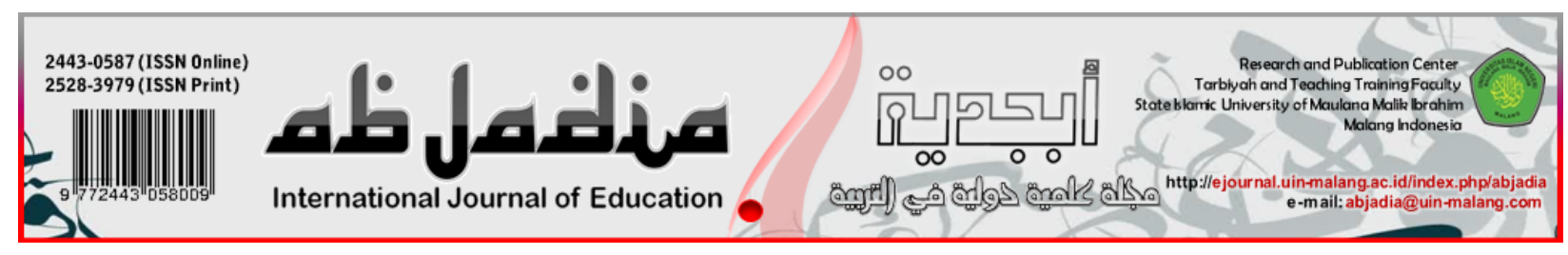

\title{
LINGUISTIC ERROR IN WRITING ARABIC ON STUDENTS OF PONDOK PESANTREN AL-MUHSININ KERINCI
}

\author{
الأخطاء اللغوية في كتابة اللغة العربية لدى طلبة معهد المحسنين الإسلامي كرينجي
}

\author{
Muhammad Fadhlan ${ }^{*}$, Uril Bahruddin, ${ }^{2}$ Ahmad Fikri ${ }^{3}$, Nur Yasin Shirotol Mustaqim ${ }^{4}$, \\ Abdul Muid ${ }^{5}$ \\ 1,2,4 Universitas Islam Negeri Maulana Malik Ibrahim Malang, ${ }^{3}$ Institute Islam Negeri Kerinci, \\ ${ }^{5}$ Universitas Jambi
}

Article History:

Received : 2021-03-19

Revised : 2021-05-20

Accepted : 2021-06-24

Published : 2021-06-30

\section{Keywords:}

Writing Skills, Linguistic Error,

Grammar, Culture.

*Correspondence Address:

fadhlanesia@gmail.com

urilbahruddin@pba.uin-malang.ac.id

fikrinurdinahmad@gmail.com

200104310013@student.uin-

malang.ac.id

abdulmuid02@unja.ac.id

\begin{abstract}
Language competence of students when learning Arabic is very little noticed by Arabic language learners because it is very difficult for students to apply to what they learn from Arabic verbally and editorially. This study aims to describe the types of linguistic errors in Arabic writing and the reason for this error among Arabic learning in al-Muhsinin boarding schools. This study uses Henry Tarigan's qualitative error analysis method which consists of four stages: identifying errors, classification of errors, explaining errors, and evaluation of errors. This study also uses Miles and Huberman method in identifying the causes of the occurrence of error, namely: observation, interview, and documentation. The Documentation used in this studies is: student notebook in translate lessons (tarjamah), student notebook about holiday. The results of this study cover several aspects, namely grammatical errors, mistakes in choosing the right Arabic vocabulary, and cultural errors in writing Arabic in accordance with the grammar in Arabic. Linguistic errors obtained are as follows: students' mistakes determine nakirah and ma'rifah, ifrod, tas'niah and jamak, irob, kalimat azzaidah and kalimat an-naqisah. The errors in the selection of the right Arabic vocabulary are: 1) Using the word "Takrir adDars" in the sentence repeating learning, 2) Using the word "Istagraqo" in timeconsuming sentences. The causes of errors in writing Arabic are: students' ability to master Arabic vocabulary is very low, lack of governance in Arabic (Nahwu and Sharaf). From some of the results presented above can be an evaluation material for Arabic learners, especially in the learning of writing skills.
\end{abstract}

$$
\begin{aligned}
& \text { تعتبر الأخطاء اللغوية أمرا طبيعيا عند تعلم اللغة الثانية واكتسابها اللغة من قبل متعلمي اللغة، } \\
& \text { ومن أسباب وقوع الأخطاء عدم معرفة علم اللغة وقواعدها.(Yoon 2012: 2) درس اللغة العربية هي درس } \\
& \text { مهم في المعاهد الإسلامية والمدارس الاسلامية حتى كانت في الجامعة الاسلامية في إندونيسيا حيث أن اللغة } \\
& \text { العربية هي لغة القرآن التي ألقى إلى الفكر الإنساني وكذلك اللغة العالمية الكبرى تحدثوا بها أكثر من مائتين } \\
& \text { مليون شخص في العالم، واستخدمها رسميا لجميع البلاد العالم العربية السعودية والجزائر }
\end{aligned}
$$


الأخرى.(Suryadarma 2016) تعليم اللغة العربية في المدارس تنقسم إلى اربع المهارات اللغوية وهي الاستماع، والكلام، والقراءة، ثم الكتابة. ومما لاشك أن تعليم مهارة الكتابة لا يدرس أكثر في المدارس لأهها درس صعب. حيث هذا الدرس يهتم إلى القواعد ومفرداتها وتعبيرها المعينة. مهارة الكتابة لها دور مهم وكبيرة التي لابد أن يستوعبهم متعلمي ومعلمي اللغة العربية في المدارس والجامعات الإسالامية وهي وسيلة الاتصال للتعبير عما يدور في النفس والخاطر.(Badruzzaman 2013: 2) تعني أنها الأسـاس الأول لفهم الكتابة العربية قواعدا وتعبيرا. وهي كذلك احدى المهارة المحتاجة في هذا العصر كالطريقة أو الوسيلة لتعبير الأفكار، والشعر، والغاية.(Jumraini: 2017: 2). ومن هذا تعتبر أن مفهم الكتابة العربية تحتاج إلى فهم مهارة الكتابة وسهل على المتحدثين أن يتواصل مع الآخرين. فالكتابة تعتبر من المهارات الإنتاجية التي تحتاج إلى استخدام المدخل الاتصالي في تحقيقها. أصبحت الكتابة أمرا بديلا عن الكلام لعدم توفر المجال للأداء الشفهي، فمهارة الكتابة تحلو مكانة مهارة الكلام فى الأهمية. في هذه المقالة يودّ الباحثون أن يقوموا بوصف أنوع الأخطاء اللغوية عند كتابة اللغة العربية لدى طلبة معهد المحسنين الاسلامي كرينجي. وحاولت الدراسة عن الإجابة عن السؤال: ما أنواع الأخطاء اللغوية من جهة اختيار المفردات، في التراكيب، وفي الثقافة. وما اسباب وقوع الأخطاء اللغوية لدى طلبة معهد المحسنين الاسامي في كتابة اللغة العربية. إن معرفة الأخطاء تفيد الأساتذة والدارسين في تنمية مهارة الكتابة وتصحيح ما يحدث فيها من الأخطاء التي تعرقل عملية الفهم والاتصال. ومن الدراسات السـابقة المتعلقة بهذه الدراسـة هي ما كتبه معلم وجايا في تحليل الأخطاء لطلبة قسم تعليم اللغة العربية.(Mualim, 2017) وتوصل الباحث إلى الأخطاء التركيبية، وأسباب الأخطاء الكلامية كعدم الأسوة اللغوية. وركزت كذلك زكية شهدى حيث أهها كتبت في موضوع تحليل الأخطاء الصهوتية في مهارة القراءة لدى طالبات الصف الثامن بمعهد إنساني Aceh Besar.A Syahda, 2020) ) وتوصل الباحثة إلى أن الطالبات تصعب في نطق الحرف العربية الصحيحة، ومن أسبابها قلة تحليل شكل الحرف والأصيوات التي تختلف عن اللغة الإندونيسية. وقام أورل بحر الدين بالبحث في تحليل الأخطاء الشفهية الشائعة لدى طلاب قسم تعليم اللغة العربية في الجامعة الإسلامية الحكومية بمالانج.(Bahruddin 2019) وتوصل الباحث إلى تلقي الأخطاء للطلبة من حيث أصهوات تكون في ابدال المد قصرا، وابدال الصوت بصوت آخر، ابدال حركة الفعل بحركة آخر من حيث مفرداتها حيث الطلبة لا يلاءم في اختيار المفردات المقررة. 


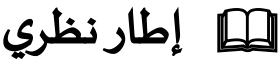

\section{مفهوم مهارة الكتابة}

مما لا شك أن الكتابة وقواعدها لا يمكن تفريقهما لأن النظام اللغوية هي الأسـاس الأول لابد الاهتمام بها نحو الطلبة في دراسة الكتابية. ؤ والكتابة هي احدى الآلة الاتصالية عند الأفراد أي نظام الاتصال عند كل فرد.(Yoandita 2019) والمهارة في اللغة هي الخذف في الشيء. والماهر: الحاذق بكل عمل، وأكثر ما يوصف السابح المجيد. والمهارة هي الحذق في الثيء والإحكام له والأداء المتقن لله. أما الكتابة هي تحويل الأصيوات اللغوية إلى الرموز مخطوطة على الورق أو غيره وهي احدى المهارات اللغوية الأربعة تحتل الكتابة مكانا شريفا في اجراء التعليم والتعلم (Badruzzaman: 2013). والكتابة أيضيا هي الوسيلة لاتصال بين الأفراد وغيره، ممن تفصله عنهم المسافات الزمانية أو المكانية (Uril, 2013). تعتبر مهارة الكتابة هي العنصر الأسـاسي الثقافة وضرورة اجتماعية لنقل الأفكار والتعبير عنها والوقوف على الأفكار ولآراء على الآخرين والإلمام بها. وفي مجال تعليم المهارات اللغوية فإن التعبير التحريري (أو مهارة الكتابة) ليست فرعا من فروع لغويا معزول عن بقية فروع اللغة. بل هي داخلة إلى مهارات اللغوية إلى حد كبير كالنحو، والصرف، والإملاء، والخط، ولإنشـاء، والترجماة. (Musthofa 2013) مهارة الكتابة هي القدرة في اخراج الآراء من الكتابة السهولة حتى الكتابة في الإنشاء(Nisa' and Ni'mah 2017: 255). إن أهداف لتعليم مهارة الكتابة هي: 1) كتابة الحروف العربية وادراك العلاقة بين شكل الحروف وصيوته، 2) كتابة الكلمات العربية بحروف منفصلة وبخروف متصلة مع تمييز شكل الحرف في أول الكلمة وأوسطها وآخرها. 3) اتقان المتابعة من اليمين إلى اليسار 4) اتقان طريقة كتابة اللغة بخط سليم ووضيحةة، 5) معرفة المبادئ الإملاء وادراك اللغة العربية نطقا، كتابة، أو عكسهما. 6) ترجمة أفكاره كتابة في الجملة والترتيب العربي مناسبة مع الكلمات.7) تعبير أفكار الكاتب في الجمل حسب الصيخ النحوية المناسبة،(Yahya 2017) 8) سرعة الكتابة وسلامتها معبرا عن نفسـة، 9) نقل الكلمات في السبورة نقلا صحيحا، 10) مراعاة الحروف المناسب حسب صيغها طولا واتساعا. (Abdul 2017) 11) قدرة الطلبة في اختيار المفردات أو الكلمة المقررة، 12) تجرية الطلبة في اخراج الآراء وتعبير اللغة العربية صحيحا ووضوحا. (Nurul: 2017) في مسك القلم الصحيح، 2) عدم الاهتمام في وضع النطة عند كتابة الحروف، 3) عدم مراعاة نظام السطر عند الكتابة، 4) عدم الاهتمام عند وضوح في كتابة الحرف.(Yusuf 2017). لمهارة الكتابة تنقسم إلى قسمين هي الإملاء والتعبير. والإملاء ينقسم إلى ثلاثة أقسم هو الإملاء المنقول، الإملاء المنظور، والإملاء الاختباري. الهدف بالإملاء المنقول هو اصلاح قدرة الطلبة في كتابة الأحرف 
والكلمة أو اصلاح كتابة الطلبة من اليمنى إلى اليسار. والهدف من الإملاء المنظور هو الأمر إلى الطلبة أن يكتب الجملة التي تمت في تعليمها. وأما الإملاء الاختباري هو قدرة الطلبة في الاستماع والحفظ ثم كتابتها في نفس الوقت. وأما تعبير ينقسم إلى قسمين هو تعبير الإنشـاء الموجه وتعبير الإنشاء. يراد بتعبير أو انشاء موجه هو يبدأ عملية الطلبة في كتابة الكلمة ثم يتطور إلى الجملة ثم الفقرة. وأما تعبير أو انشاء اعطاء فرصة الطلبة في اختيار الموضوع ثم يطور الطلبة حتى تصبح الجملة الفقرات الكثيرة أما في اللغة العربية أو في اللغة الأم.(Hastang 2019)

\section{تحليل الأخطاء}

Pit S. الخطأ اللغوي هو الانحراف عن القواعد اللغة عن تعليم اللغة الثانية. رأى بيت كوردر Corder الخطاء هو الانحراف المنهجي والمتسق لمتعلمي اللغة في مرحلة معينة.(Daniel: 2004). ورأى طعيمة الخطأ اللغوي هو اللغة التي يستخدمها متعلمي اللغة مخالفا بقواعد اللغوية المعينة. (Thaimah: 1999). وأما الخطأ في اللغة الإنجليزية هو تقابل كلمة Error أو Mistake، حيث هناك بعض العلماء يعبر أنهما في المصطلحين واحد. والأخطاء هو جمع من الخطأ بمعنى الذنب والغلط أوضد الصيواب. عند الاصطلاح الخطأ هو يتحور حول خرق القاعدة الموضوع عليها في مجال معين، وكان الخطأ والغلط بمعنى واحد. والأخطاء هو جمع من الخطأ بمعنى الذنب والغلط أو ضيد الصيواب. عند الاصطلاح الخطأ هو يتحور حول خرق القاعدة الموضوع عليها في مجال معين، وكان الخطأ والغلط بمعنى واحد.(Bahruddin 2019) وأما تحليل الأخطاء هو عملية أو دراسة في بحث الأخطاء عند متعلمي اللغة الثانية. (Daniel:2004) رأى ريتشار (Rischard) إن تحليل الأخطاء هو البحث عن الأخطاء عند التركيب وترتيب في اللغة الثانية. (Richard: 1974). وتفريق الأخطاء ليس أمرسهلا رأى (Rischard) إن تصنيف الأخطاء ينقسم إلى قسمين هو الأخطاء في داخل اللغة هذا الأخطاء يبدأ من لسان الأم القسم الثاني هو الأخطاء داخل اللغة وتطويرها وقع هذا الأخطاء عند تعليم اللغة الثانية.(Alahmadi 2014)

من بيان السابق نستنتج أن الأخطاء اللغوية يسبب إلى انقطاع الاتصال بين الأفراد. والاتصال في اللغة هو الارتباط أو ضيد الانقطاع. وأما اللغة هي أصيوات يعبر بها كل قوم عن أغراضهه. (Guylani:2004) من تعريف السابق نعرف أن اللغة هي آلة الاتصال بين أفراد قوم وهي ليس مجرد شفهيا بل كان تحريريا. معروف لدينا سـابقا أن المشكلات التي تواجاه في تعليم اللغة العربية هي المشكلات اللغوية والمشكلات غير اللغوية. والمشكلات اللغوية هي المشكلات المتعلقة بأمر لغوي كالمفردات والقواعد والتراكيب الجملة والتعريب.(Munip n.d.) والمشكلات غير اللغوية هي المشكلات المتعلقة بالأمور الاجتماعية 
والثقافية.)(Hasani: 2017) والأخطاء الاتصالية نفسها عند تعليم اللغة العربية مدلول إلى تلك الأخطاء المذكورة. والأخطاء الاتصالية نفسها عند تعليم اللغة العربية مدلول إلى تلك الأخطاء المذكورة. الأخطاء التركيبية هي الأخطاء المتعلقة بالمشكلات اللغوية. يراد بالجانب اللغوي هنا جوانبا لغوية تشمل على المستويات الصرفية، والنحوية، والدلالية. والأخطاء الصرفية هي الأخطاء اللغويات تُدرس فياء أشكال الكلمات وتغيُرات أشكالها والمعاني التي تنمو من خلال حدوث هذه المتغيرات. أو ذلك الفرع الذي يهتم بشأن تراكيب الكلمات من حيث قواعدها. الأخطاء النحوية هي الأخطاء في وضع الكلمات مجتمعة حتى تصبح جملة مفيدة. وهو بالمعنى النظري الخلافات اللغوية من حيث الجوانب الصيوتية، والصرفية، والدلالية بين لغات الأعاجم واللغات الأم (في اللغة العربية والإندونيسية) التي تسبب إلى صعوبات الطلاب في دراسة اللغة العربية. وأما الأخطاء الدلالية هي الأخطاء المتعلقة بالتواصل بين الرمز (Form) بمعنى Grammatical (أو المعنى من ذلك الرمز. وهي ثلاث مراحل (Meaning) (Munip n.d.).(Contextual Meaning) معنى المقامي (Meaning تستمد اللغة العربية أهمية كبيرة من خلال أنها لغة الثقافة والدين والتعارف لكثير من المسلمين وغيرهم في جميع أنحاء العالم، من المسلم باه أن اللغة هي الوسيلة الوحيدة التي تسجل بها الأمة علومها، وثقافتها، وتود بها أدبها. وتكتب تاريخها ماضياه وحاضره. أما أهمية اللغة العربية تتكون من أهمية اجتماعية التي يتم من خلال التواصل مع الآخرين في المجتمع. وأهمية عقلية التي تعني بأهها أداة التفكير والثقافة للفرد. وأهمية النفسية حيث يعبر بها الإنسان عن رغباته انفعالاته باللغة العربية، مثل ذلك الشعراء، والأدباء الذين يتغنون باللغة العربية. وأهمية جمالية وهي تفيد الإنسان في تذوق الأعمال الأدبية المختلفة من شعر ونثر العربي.(Hasan: 2017). إن الثقافة اللغة العربية أحيانا تبدأ من الفعل ثم الفاعل، (S+P)، وهذه تخالف مع الثقافة اللغة الإندونيسية. تبدأ من الفاعل ثم الفعل (S+P). (Supriyanto:2018).

استخدم المدخل الكيفي في جمع البيانات نحو هذا البحث. واستخدم منهج تحليل الأخطاء لهنري تارغن في تحليل الأخطاء كتابة الطلبة في ترجمة اللغة الإندونيسية إلى اللغة العربية وهو يتكون من أربع مراحل هي: التعرف على الأخطاء، وتصنيف الأخطاء، شرح الأخطاء، ثم تقويم الأخطاء. (Tarigan: 2011:31) واستخدم كذلك طريقة ملس هبرمان لوصف اسباب وقوع الأخطاء في كتابة اللغة العربية. وهو يتكون من ثلاث مراحل هي: الملاحظة، المقابلة، والتوثيق. (Sugiyono: 2019: 233). 


\section{زآiْi نتائج ومناقشة}

\section{الأخطاء التركيبية}

انطلاقا من ورقة الطلبة وجد الباحث الأخطاء التركيبية عند كتابة الطلبة ومن الأخطاء الذين وقع الطلبة فيها وهي تتكون فيما يلي:

وقع الطلبة الأخطاء في التعريف والتنكير فيضعونها التعريف (ال) في محلّ خاطئ ظنا أن هذا هو

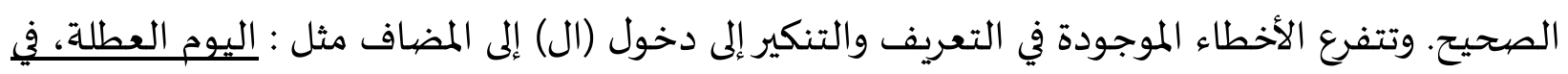

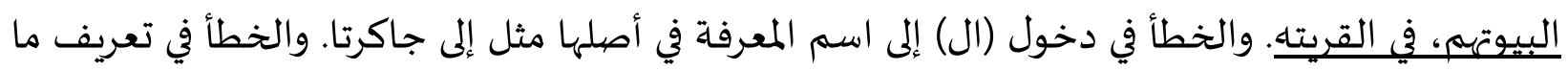

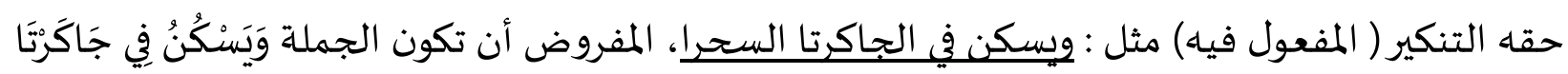
شَهْرًا وَاحِدًا. والخطأ الذي ارتكبه الطلاب أيضا في حذف (ال) ما حقها بالتعريف لمنصببه في الإعراب من التابع (النعت) مثل أسبوع الآتي.

لمزيد من التوضيح في البيان السابق يمكن اظهار تصنيف الأخطاء التركيبية في الجدول التالي:

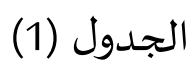

تصنيف الأخطاء الكتابية في التعريف والتنكير التيرو

\begin{tabular}{|c|c|c|c|}
\hline الصواب & 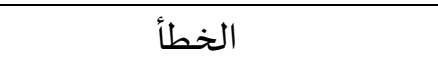 & وصف الخطأ & نوع الخطأ \\
\hline يَوْمُ العُطُطَلَةُ & اليَوْمُ العُطُلَةُ & دخول (ال) إلى المضاف & \multirow{9}{*}{ والتنكيف } \\
\hline فِي بُيُوْتهِهن & فِي البُبُوْتهِهْ & & \\
\hline إِلَى قَرَنَتِبهِ & إلِّلَ القَقَيَتَته & & \\
\hline إِالَى بَيْتِهِ & 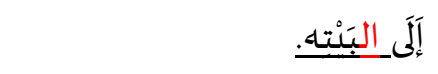 & & \\
\hline إِآلَّ جَاكَرْتَا & 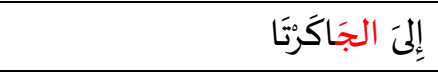 & دخول (ال) إلى أسماء المعرفة & \\
\hline إِالِّ السَّاحِلِ & 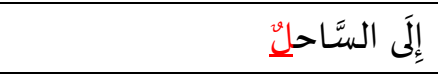 & تنوين الاسم المحلى بال. & \\
\hline أَحْمَدُ & أَحْمَدِّ & تنوين الاسم غير المنصرف & \\
\hline وَتَسنكُكُنُ فِي جَاكَرْتًَا شَهْرًا. & 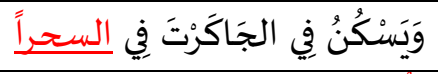 & تعريف ما حقه التنكير (المفعول فيه) & \\
\hline الأُسْبُْوُْعُ الآتِتي & أَسْبُْوُْعُ الآتِتي & حذف (ال) ما حقه بالتعريف & \\
\hline
\end{tabular}

الجملة الإسمية في اللغة العربية لابد أن تتناسب وحدة الكلمات من حيث العدد بين المبتدأ والخبر.

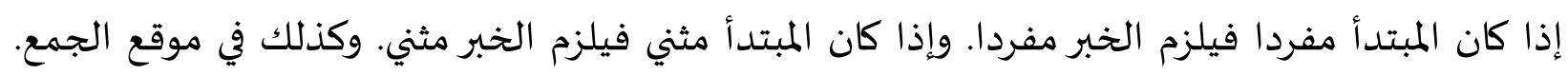

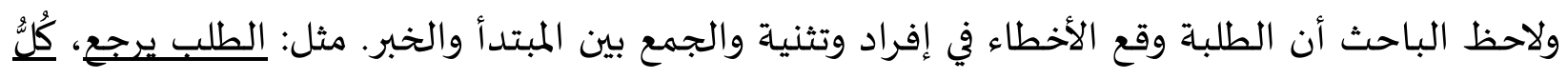

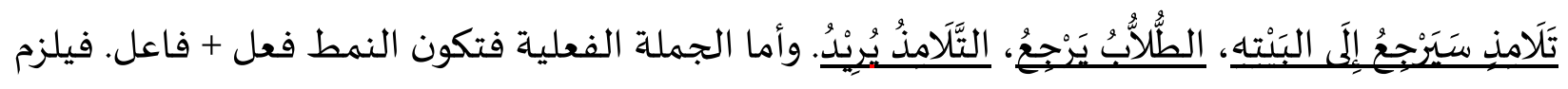


الفعل أن يكون مفردا في أول الجملة. وفاعله لا يلزم أن يكون مفردا أو تثنية وجمعا بمعني لا يلزم ملائمة بين الفعل والفاعل من حيث العدد قد يكون الفاعل جمعا ولكن الفعل لابد من مفرده. ولاحظ الباحث أن الطلبة يرتكبون الخطأ في ملائمة بين الفعل والفاعل والجملة الفعلية. مثل: سيرجعون الطلاب. لمزيد من التوضيح في البيان السابق يمكن اظهار تصنيف الأخطاء التركيبية في الجدول التالي:

الجدول (2)

تصنيف الأخطاء الكتابية في الإفراد والتثنية والجمع

\begin{tabular}{|c|c|c|c|}
\hline الصواب & 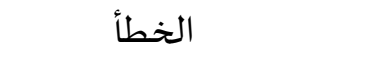 & وصف الخطأ & نوع الخطأ \\
\hline الطُلَّابُ يَرْجِعُوْنَ. & الطُّلَبُ يَزْجِعُ & عدم التناسب بين المبتدأ & الإفراد والتثنية \\
\hline كُلُّ تِلْمِيْذٍِ سَيَرْجِ & 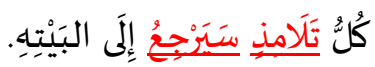 & والخبر في الجمع & والجمع \\
\hline سَيَرْجِعُ الطَّلَّبُ & 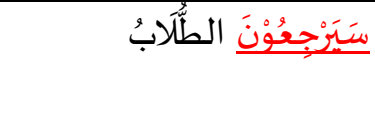 & الجملة الفعلئة بين الفعل والفاعل في & \\
\hline
\end{tabular}

تتمز اللغة العربية بإعراب الكلمة في الجملة. موضع الكلمة في الجملة يعين شكل أواخر الكلمة. الإعراب ينقسم إلى أربعة منها: الرفع، والجر، والنصب، والجزم. وكل إعراب له أبواب مثل الرفح بالضماة، بالآلف، والواو، والجر بالكسرة، والفتح، والياء، والنصب بالفتح، والكسر وخذف النون، والجزم بالسكون، وحذف حرف العلة والنون. وهذه علامات الاعراب تعتمد على موضع الكلمات في الجملة. كالمبتدأ حقه بالرفح، والمفعول باه حقه بالنصب وهلم جرا.

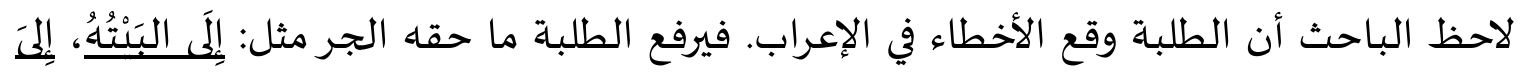

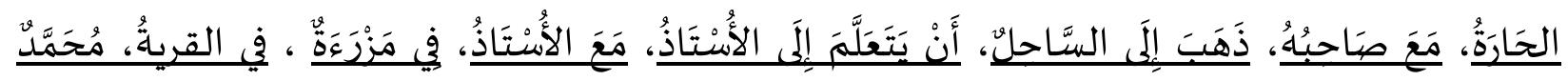

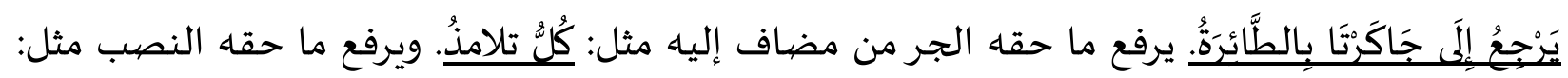

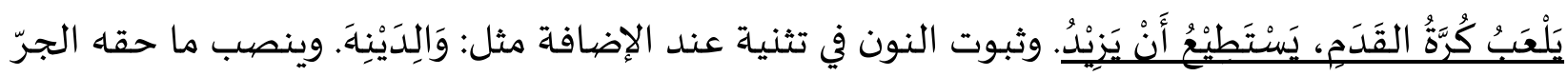

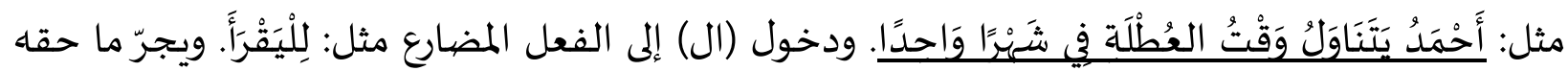
النصب مثل: لِيُكَرِّرِ الدَّْرَسَ.

لمزيد من التوضيح في البيان السابق يمكن اظهار تصنيف الأخطاء التركيبية في الجدول التالي: 
(3) الجدول)

تصنيف الأخطاء الكتابية في الإعراب

\begin{tabular}{|c|c|c|c|}
\hline الصواب & 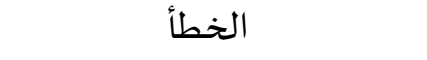 & وصف الخطأ & نوع الخطأ \\
\hline إِلَن بَيْتِتِك & إِلَى البَيَتِتُهُ & رفع ما حقه الجرّ & الإعراب \\
\hline إِلَى الحَارَة & إلى الَِّ الحَارَةُ & & \\
\hline مَعَ صَاحِبِدِ & مَعَ صَاحِبُْهُ & & \\
\hline كُلَّ تِلْمِيْذٍِ & كُلٌّ تلامذُّ & رفع ما حقه الجرّ (مضاف إليه) & \\
\hline يَلْعَبُ كُرَّةَ القَدَمِ & يَلْعَبُ كُرَّةُ القَدَدَمِ & رفع ما حقه النصب & \\
\hline يَسنتَطِيْعُ أَنْ يَنِيْدَ & يَسنتَطِيْعُ أَنْ يَنِنْدُ & & \\
\hline \multirow[t]{2}{*}{ وَالِدَديْهِ } & 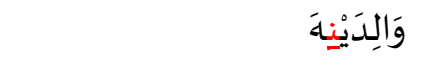 & ثبوت النون في تثنية عند & \\
\hline & & الإضافة & \\
\hline أَحْمَدُ يَقْضِي العُطُلََْ فِي شَهْرِ & أَحْمَدُ يَتَنَاوَلْ وَقْتُ العُطُلَْة فِيْ & نصب ما حقه الجرّ & \\
\hline وَاحِدٍ & شَهْرًا وَاحدًا & & \\
\hline لِيَقْرَأَ & لَلْيَقَرَأَ & دخول (ال) إلى الفعل المضارع & \\
\hline ل لِيَكَرِّرَ الدَّرْسَ & ل لِيُكَِِّر الدَّرَسنَ & جرّ ما حقه النصب & \\
\hline
\end{tabular}

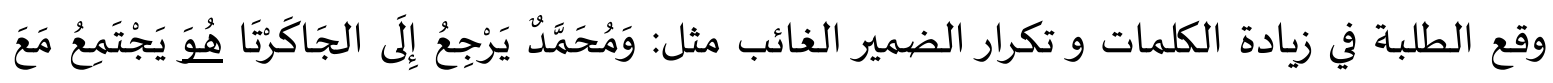
صَاحِبَاُ، في الجملة واستخدام الحرف في محل خاطئ. مثل دخول حرف إن إلى فعل المضارع: لأَنْْ يَنْسُرَ

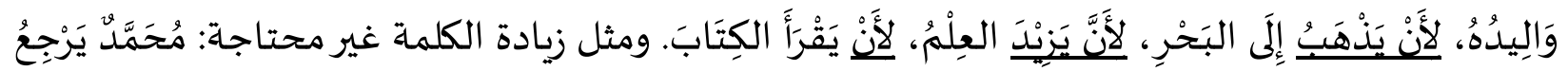

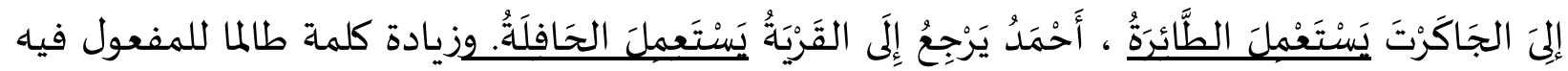

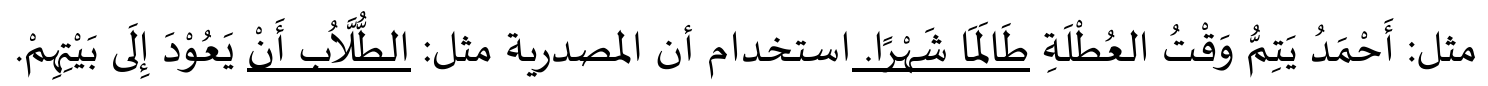
لمزيد من التوضيح في البيان السابق يمكن اظهار تصنيف الأخطاء التركيبية في الجدول التالي:

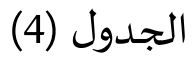

تصنيف الأخطاء الكتابية الكلمة الزائدة

\begin{tabular}{|c|c|c|c|}
\hline الصواب & الخطأ & وصف الخطأ & نوع الخطأ \\
\hline 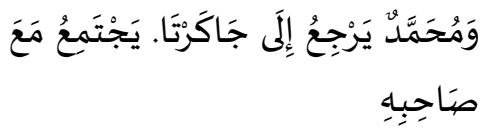 & 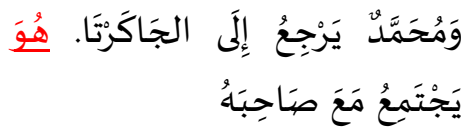 & تكرار ضيمير الغائب & الكلمة الزائدة \\
\hline 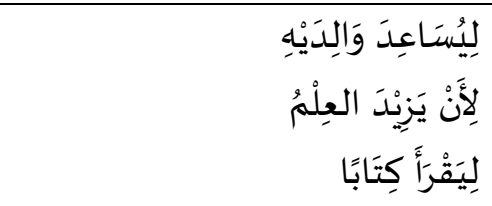 & 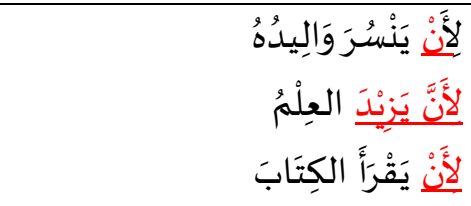 & الفعل المضيارع حرف إن إلى & \\
\hline
\end{tabular}




\begin{tabular}{|c|c|c|}
\hline مُحَمَدُّ إِلَى جَاكَرْتَا بِاطَّأَيَرَة & 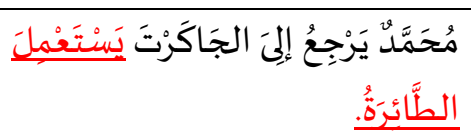 & محتادة الكلمة غير \\
\hline أَحْمَمَدُ يَتِهُ وَقَتُ العُطُلْةِة شَهْرًا & 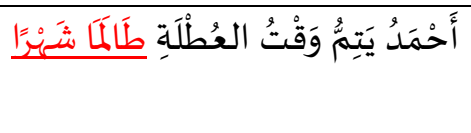 & للمفادة كلمة طالما \\
\hline 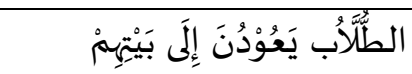 & 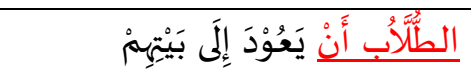 & استخدام أن المصدرية \\
\hline
\end{tabular}

وقع الطلبة في وضع الكلمات كعدم وضع حرف أن المصدرية بين الفعلين المضارعين. مثل: يُرِيْدُ

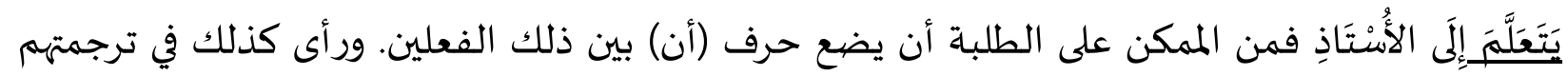

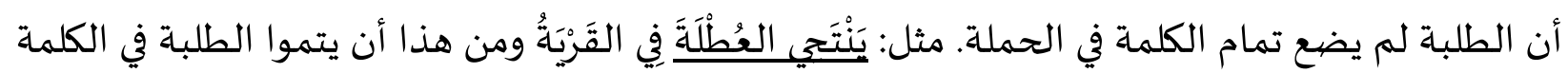
الصحيحة عند وضع الكلمة. ولاحظ الباحث كذلك. أن الطلبة لم يعرف المفردات على وجه مشمل. مثل:

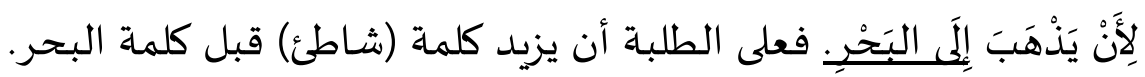
لمزيد التوضيح من اليانات السابقة يمكن أن ننظر إلى الجدول التالي:

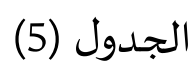

تصنيف الأخطاء الكتابية في الكلمة الناقصية

\begin{tabular}{|c|c|c|c|}
\hline الصواب & الخطأ & وصف الخطأ & نوع الخطأ \\
\hline 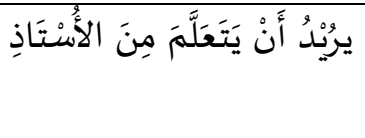 & 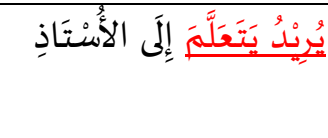 & الفعلين حرف المضارعين المصدرية بين & الناقصة \\
\hline 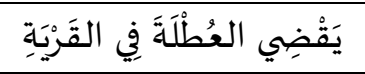 & يَنْنَحِي العُطُلْنَ فِي القَرْيَّةُ & وضيح الكلمة غير التامة & \\
\hline 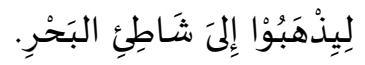 & لَالَنَنْ يَذْهَبَ إِلَى الْبَحْرِ & التنبؤ نحو المفردات ناقصة & \\
\hline
\end{tabular}

وقع الطلبة الأخطاء في تعيين تنوين في فعل المضارع. حيث أن الطلبة يعين تنوين في فعل المضارع

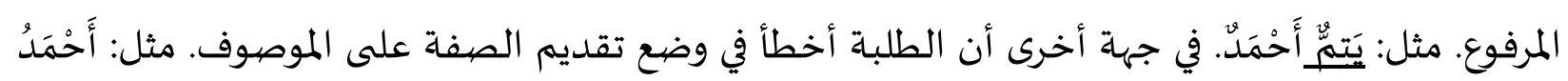

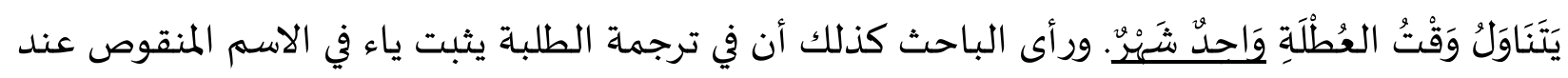
التنكير. مثل:في أُسْبُوْغِع آتي.

لمزيد من التوضيح في البيان السابق يمكن اظهار تصنيف الأخطاء التركيبية في الجدول التالي: 
(6) الجدول

تصنيف الأخطاء الكتابية النحوية الأخرى

\begin{tabular}{|c|c|c|c|}
\hline الصواب & الخطأ & وصف الخطأ & نوع الخطأ \\
\hline يَتِتهُ أَحْمَدُ & يَتَعْ أَحْمَمَد & تنوين فعل المضارع & أخطاء نحوية أخري \\
\hline أَحْمَدُ يَتَنَاوَلُ وَقْتُ العُطْلَةِة شَهْرُ & أَحْمَدُ يَتَنَاوَلُ وَقْتُ العُطْلَةِة & تقديم الصفة على & \\
\hline وَاحِدْ & وَاحِد شَهْرِّن & الموصيوف & \\
\hline فِي أُسْبُوْغِعٍ آتٍ & فِي أُسْبْوْغِعٍ آَتَي & ثبوت ياء في الإسمى & \\
\hline
\end{tabular}

الأخطاء في اختيار المفردات

انطلاقا من كتابة الطلاب في ورقة الطلبة وجد الباحث الأخطاء في اختيار المفردات الجيدة عند ترجمة اللغة الإندونيسية إلى اللغة العربية ومن الأخطاء التي يرتكب الطلاب فيما يلي:

Dan akan menghabiskan liburan di kampung وقع الطلبة الأخطاء في اختيار المفردات في جملة حيث يستخدم الطلاب كلمة: يتناول العطلة في القرية، ويته العطلة في القرية، وأن ينتهي_العطلة في القرية. وسنتحي العطلةَ في القريةُ، سينتهي في القرية، واستغرق العطلة في الحارةٌ. والكلمة اللازمة في هذه الجملة هي: ويقضي العطلة في القريـة. وقع الطلبة الأخطاء في اختيار المفردات عند ترجمة اللغة الإندونيسية إلى اللغة العربية في الجملة. (Dia ingin belajar kepada Ustadz untuk mengulangi pelajaran) "Mengulangi Pelajaran" في الجملة بكلمة ( ليكرر، للرجعا، يعيد، لأن ذاجع، ليراجع، للمراجعة). المثال: يريد أن يتعلم إلى الأستاذ ليعيد الدرس، هو يريد يتعلم إلى الأستاذ للمراجعة الدرس، سيتعلم مع الأستاذ لـراجع الدرس، هو يريد أن يتعلم مع الأستاذ لأن ذاجع، يريد أن يدرسن مع الأستاذ للرجعا الدرس، هو يزيد أن يتعلم إلى الأستاذُ ليكر الدرس. إن ترجمة المعمدة في ترجمة هذه الكلمة حسب ثقافة العربية هي (المذاكرة/ ليذاكر). وينبغي على الطلبة أن يترجم هذه الجملة بالترجمة الآتية: ويريد أن يتعلم إلى الأستاذ لمذاكرة الدرس. لمزيد التوضيح من الأخطاء في اختيار المفردات سابقا يمكن تصنيف الأخطاء في الجدوال التالي: 


$$
\text { (7) (7) (الجدول) }
$$

تصنيف الأخطاء الكتابية في اختيار المفردات

\begin{tabular}{|c|c|c|}
\hline الصواب & الأخطاء & النص \\
\hline ويقضي العطلة في القرية. & 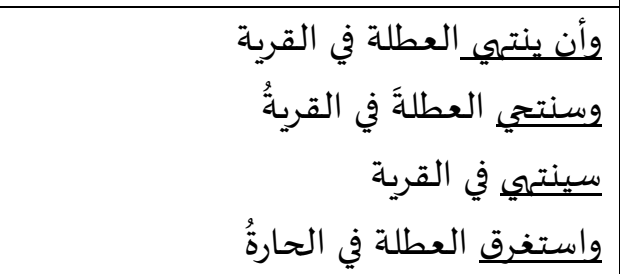 & $\begin{array}{l}\text { Dia akan Menghabiskan } \\
\text { waktu liburan di } \\
\text { Kampung }\end{array}$ \\
\hline لمذاكرة الدرس. يتعلم إلى الأستاذ & 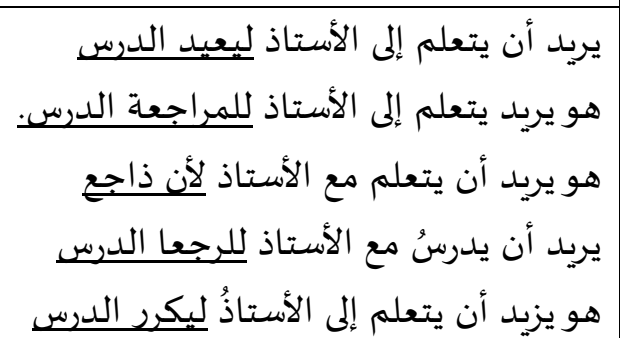 & $\begin{array}{l}\text { Dia ingin belajar kepada } \\
\text { Ustadz untuk mengulangi } \\
\text { pelajaran. }\end{array}$ \\
\hline
\end{tabular}

\section{الأخطاء الثقافية في كتابة اللغة العربية}

وقع الطلبة في الثقافة العربية عند كتابة اللغة العربية. اخطؤوا الطلاب في ترجمة الجملة Sedangkan ahmad kembali ke kampung menggunakan bis. حسب ثقافة اللغة العربية عند كتابة اللغة العربية. المثال: إذا أحمد برجع إلى القرية بالحافلة، إذن أحمدِ ييعتي إلى القريته استعمل اتوبس، بل أحمد إلى القرية بالحافة، ولكن احمد سيرجع إلى القريتاء بالئتوبلس. إن بعض الطلبة يزيد حرف (إذا، إذن، بل، لكن) في الجملة. مع أن هذه الزيادة غير محتاجة في ترجمة تلك الكلمة. ووجد الباحث كذلك أن بعض الطلبة يترجم كلمة "Bis" بكلمة (اتوبيس، اتوبس)، مع أن هذه الترجمة يشترك النمط الإندونيسيا. المثال: إذن أحمد ييعتي إلى القريته استعمل اتوبس، ولكن احمد سيرجع إلى القريته بالئتوبلس. إن الترجمة المتعمدة بالثقافة العربية عند ترجمة كلمة" Bis" هي (الحافلة). وينبغي على الطلبة أن يترجم الجملة بالآتية: ويسافر أحمد إلى القرية بالحافلة. وقع الطلبة الأخطاء في تعيين شباه الجملة (prasa) المثال: (غدا هو الوقت العطلة/ في يوم الغدهو يوم العطلة). حيث أن الطلبة يزيد ضمير "هو" في الكلمة. مع أن هذا الضمير لا يحتاج في تلك الكلمة. وفي المثال الآخر: يترجم الطلبة كلمة (Besok adalah hari libur)، يوم الغاد العطلة. ينبخي في هذه الكلمة أن يستخدم الطلبة على شكل شبه الجملة أو الظرف. والكتابة المقررة في هذه الجملة هي: (غدا يوم العطلة// 
إن ترتيب الكلمة في اللغة العربية يخالف مع الترتيب في اللغة الإندونيسية. إن الكلمة في اللغة

العربية تبدأ بالفعل(Predikate). وأما اللغة الإندونيسية عامة تبدأ بالفعل - الفاعل(Subjek + Predikate) وقع بعض الطلبة الأخطاء في وضع الجملة المقررة حسب ثقافة اللغة العربية. اخطؤوا الطلبة في ترجمة (Para siswa akan kembali kerumah mereka minggu depan) الآتي/ كُلُّ تَلَميْذُ برجع إلى البيته في أسبوع الغاد). والجملة المقررة هي: سـسـافر الطلاب إلى بيتهم بعد

لمزيد التوضيح من الأخطاء الثقافية سابقا يمكن تصنيف الأخطاء في الجدوال التالي:

(8) (8) (8) (1) (1)

تصنبف الأخطاء الكتابية في الثقافة العربية

\begin{tabular}{|c|c|c|}
\hline وجوه الصواب & وجوه الأخطاء & النص ال النص \\
\hline ويساف أحمد إلى القرية بالحافلة. & 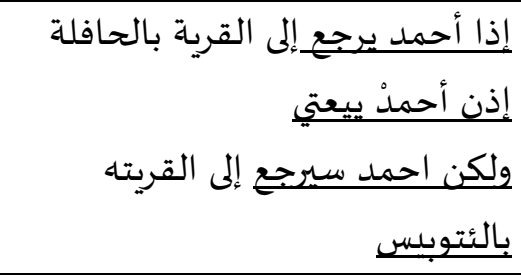 & $\begin{array}{l}\text { Sedangkan ahmad kembali } \\
\text { ke kampung menggunakan } \\
\text { bis. }\end{array}$ \\
\hline غدا يوم العطلة/ الإجازة. & غيدا هو الوقت العطلة/ في يوم يوم الغياد العطلة & Besok adalah hari libur \\
\hline الأسبوع. سيسافر الطلاب إلى بيتهم بعد & 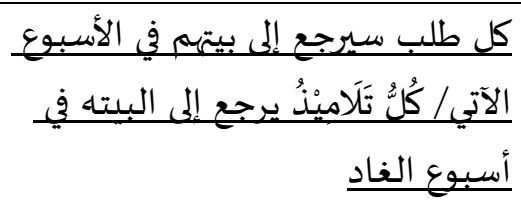 & $\begin{array}{l}\text { Para siswa akan kembali } \\
\text { kerumah mereka minggu } \\
\text { depan }\end{array}$ \\
\hline
\end{tabular}

\section{تقويم الأخطاء في كتابة اللغة العربية لدى طلبة معهد المحسنين الاسلامي}

يتبين من البيانات السابقة أن نسبة الأخطاء التركيبية لدى طلبة معهد المحسنين الاسلامي هي 40 \% ومن هذا أن كفاءات الطلبة في استيعاب التركيب عند كتابة اللغة العربية لم يكن جيدا بسبب عدم فهم القواعد جيدا. وأما الملائمة نحو اختيار المفردات الصحيحة والاهتمام نحو ثقافة اللغة العربية المقررة عند كتابة اللغة العربية في ترجمة اللغة الاندونيسية إلى اللغة العربية أن نسبة الطلبة في 40 \% ومن هذا أن استيعاب الطلبة نحو المفردات والاهتمام نحو ثقافة اللغة العربية يقوم في درجة جيد. وأما الكتابة الصحيحية بنظر إلى جهة اختيار المفردات، والتراكيب، والثقافة اللغة العربية تقوم في درجة 20\%. ومن هذا نستنتج أن الأخطاء في كتابة اللغة العربية لدى طلبة معهد المحسنين في درجة ضعيفة. 


\section{اسباب وقوع الطلبة في أخطاء كتابة اللغة العربية}

\section{قلة استيعاب المفردات للغة العربية}

إن هدف تعليم اللغة العربية في معهد المحسنين هو لتركية كفاءة اللغة لدى الطلبة ولفهم كتب التراث العربية. إن قلة استيعاب المفردات نحو الطلبة يسبب إلى عدم اعطاء المفردات كل يوم وعدم همئم الاهتمام في مهارة الكلام حتى يصعب على الطلبة أن يضع المفردات المقررة في الجملة المفيدة عند

$$
\text { كتابة اللغة العربية وبالخصوص في دروس الترجمة. }
$$

\section{علدم الاهتمام في القواعل النحوية والصرفية.}

الاستيعاب نحو القواعد اللغوية كالنحو والصرف في تعليم مهارة الكتابة أمر مهم، لأهها الأساس الأول في تعليم مهارة الكتابة، (Royani and Sadiah 2019: 764). استخدم معهد المحسنين الطريقة القواعد في تعليم القواعد النحوية حيث الأستاذ لا تأمر الطلبة بوضع الجملة الكثيرة المتعلقة بالقواعد النحوية والصرفية. ومن هنا يصعب الطلبة أن يضع الجملة حسب القواعد النحوية والصرفية.

\section{علدم استخدام القاموس أو المعجم المقرة لبحث المفردات المقررة.}

إن مدرسي اللغة العربية لا يهتم إلى استخدام القاموس والمعجم في تعليم اللغة العربية. حيث الطلبة لا يستطيع أن يعين المفردات المقررة في ترجمة اللغة الإندونيسية إلى اللغة العربية. انطلاقا من البيانات السابقة توصل الباحث أن هناك اسباب وقوع الطلبة في الأخطاء الكتابية وهي عدم الاهتمام في القواعد النحوية والصرفية وهذا السبب يسبب إلى قلة معرة الطلبة في ترجمة اللغة العربية لأن الترجماة تحتاج إلى الاهتمام بالقواعد الصحيحاة. بجانب الاهتمام إلى القواعد الجيدة يمكن أن يعطى المدرس المفردات المقررة المستخدمة لدى قوم العرب لأن كثرة المفردات تؤدى إلى سهولة وضع

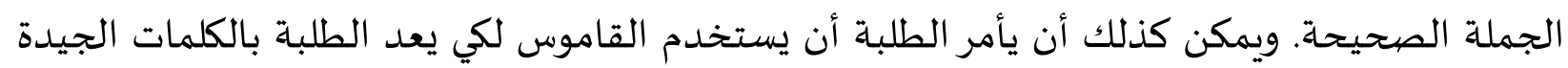
خصوصا في تعليم مهارة الكتابة..

\section{خاتمة 㴘}

بعد انتهاء في عملية تحليل البيانات في كتابة اللغة العبية لدى طلبة معهد المحسنين الاسلامي كرنيجى يستخلص هذا البحث بالأمور التالية: أن هناك اربع انواع في الأخطاء الكتابية لدى طلبة معهد المحسنين

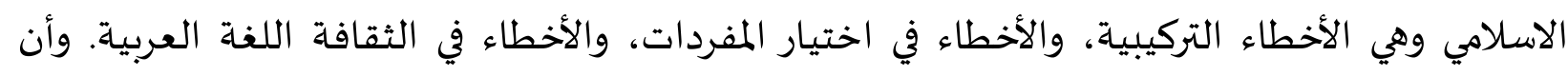
الأخطاء التركيبية لدى طلبة معهد محسنين في كتابة اللغة العربية هي: من حيث التعريف والتنكير، الإفراد 
والتثنية والجمع، الإعراب، الكلمة الزائدة، والكلمة الناقصة. وأما الأخطاء من الجهة اختيار المفردات هي حيث الطلبة لا يهتم المفردات المناسبة في كتابة اللغة العربية عند ترجمة اللغة الإندونيسية إلى اللغة العبية. ومن جهة الثقافة للغة العربية حيث الطلبة لايهتم الجملة المقررة المستخدمة في اللغة العربية الصحيحة. أن بعض الطلبة يستخدم الترتيب الإندونيسية في كتابة اللغة العربية.

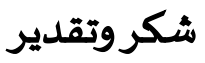

جزيل الشكر إلى كلية انسـانية بجامعة مولانا مالك إبراهيم الإسلامية الحكومية مالانج على اعطاء الفرصة الغالية حتى يتم كتابة هذا البحث.

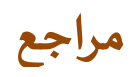

Al-Ghulayni, Mustofa. (2004). Jami'u Al-Durus Al-Arabiyah. Beirut: Al-Ashriyyah.

Al-Hasani, Al-Ba'u. (2017). Al-Musykilatu Al-Lughawiyyatu Fi Al-Tarjamati Bahsun Bi Al-Jami'ati Al-Islamiyyati Al-Hukumiyyati Ponorogo, Al-Majallatu Al-Ilmiyyatu Al-Arabiyyu, Al-Mujalladu Al-Awwalu.

Badruzzaman. (2013). تعليم مهارة الكتابة لغير ناطقين بغيرها. DIDAKTIKA, Vol.XVI

Bahrudin, Uril. (2013). Tathwiru Manhaji Ta'limi Al-Lughah Al-Arabiyah Wa Tathbiqihi 'Ala Maharati Al-Itabati. Malang: UIN Maliki Press.

Fadlillah, Nurul. (n.d.). Al Kalimah Al Mutaqati'ah Sebagai Media Permainan Bahasa Arab dalam Maharah Kitabah.

Fgoni, Djunaidi, and Almanshur. Fauzan. (2012). Metode Penelitian Kuantitatif, cet. 1, Jogjakarta: Ar-Ruzz Media.

Hasan, Nur. Ahammiyyatu Al-Lughati Al-Arabiyyati 'Ala Tathawwuri Al-Lughati Wa Al-Tsaqafati Al-Indonesiyyati', Al-Multaqa Al-Alamiyyatu Al-Tsaniyya 'Asyara Li AlLughati Al-'Arabiyyati.

Laila Munirah, Abdul Qadir. (2017). Tahlilu Al-Akhtha'i Al-Imlaaiyyati Fi Kitabati AlThullabi Fi Al-Mustawaa Al-Rabi'i, Al-Tadris: Jurnal Pendidikan Bahasa Arab, 5.1 https://doi.org/10.21274/tadris..5.1.21-42

Longman, Suryadarma, Yoke. (2016). Al-Tadribat Al-Lughowiyah Al-Musyawwiqat Fi Ta'limi Al-Lughah, Lisanudhad, 3.2 10, <https:/ / ejournal.unida.gontor.ac.id/index.php/lisanu/article/view/1107>.

Munip, A. (n.d.). Problematika Penerjemahan Bahasa Arab ke Bahasa Indonesia ; Suatu Pendekatan Error Analysis. Al-'Arabiyah, Vol. 1(No. 2), Retrieved from http:/ / digilib.uin-suka.ac.id/8008 
Nesreen, Alahmadi. (2014). Errors Analysis: A Case Study of Saudi Learner's English Grammatical Speaking Errors, Arab World English Journal, 5.4.

Nisa', D. K., \& Ni'mah, J. (2017). Pengembangan Bahan Ajar Mahārah Al-Kitābah Berbasis Pendidikan Karakter bagi Mahasiswa Program Studi Pendidikan Bahasa Arab STAI Attanwir Bojonegoro. Jurnal Al Bayan: Jurnal Jurusan Pendidikan Bahasa Arab, 9(1). https://doi.org/10.24042/albayan.v9i1.1241

Parera, Jos Daniel. (2004). Linguistik Edukasional, Jakarta: Erlangga.

Richard, Jack C. Error Analysis: Perspectives an Second Language Acquisition, London. (1974).

Royani, S., \& Sadiah, S. (2019). An Analysis of Grammatical Errors in Students' Writing Descriptive Text. PROJECT (Professional Journal of English Education) https:/ / doi.org/10.22460/project.v2i6.p764-770

Sugiyono. (2014). Metode Penelitian Kuantitatif Kualitatif Dan R E D. Bandung, Alfabeta. Supriyanto, Dedi. Pendidikan Bahasa PPs UNJ, Perbandingan Struktur Klausa Bahasa Arab Dan Bahasa Indonesia.

Syahda, Z., Muslim, B., \& Safariah, S. (2021). Tahlil Al-akhto' as-Sawtiyyah FiMaharatil-Qiro'ah lada-Thoolibat as-Shoffu ast'min bi-Ma'had Insaniy Qur'aniy Aceh Besar (LISANUNA): Jurnal Ilmu Bahasa Arab Dan Pembelajarannya, 10(2), 22. https://doi.org/10.22373/ls.v10i2.8826

Tarigan, Henry Guntur \& Tarigan Djago. (2011) Pengajaran Analisis Keselahan Bebasis Keselahan Berbahasa, Bandung: Angkasa.

Thaimah, Rusydi Ahmad. (1999) Al-Ta'limu Al-Arabiyatu Lighairi Al-Nathiqina Biha, Mesir: Issaqa.

Wijaya Muallim. (2017). Error Analysis for Students of the Department of Arabic Language Teaching. IJ-ATL (International Journal of Arabic Teaching and Learning) Vol. 01.

Yoandita, P. E. (2019). An Analysis of Students' Ability and Difficulties in Writing Descriptive Text. Jurnal JOEPALLT (Journal of English Pedagogy, Linguistics, Literature, and Teaching) https://doi.org/10.35194/jj.v7i1.53

Yoon, H. (2012). Grammar errors in Korean EFL learners' TOEIC speaking test. English Teaching.

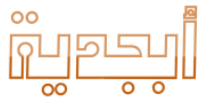

